\title{
A Novel Energy Harvesting Scheme for Mixed FSO-RF Relaying Systems
}

\author{
Jianchao Chen, Liang Yang, Wenjie Wang, Hongchuan Yang, Yuanwei Liu, Mazen O. \\ Hasna, Mohamed-Slim Alouini
}

\begin{abstract}
In this paper, we consider the performance of a mixed free-space optical and radio frequency (FSO-RF) relaying system with energy harvesting in the existence of both atmospheric turbulence and pointing errors. More specially, we assume that the direct current (DC) component of the FSO signal, which is generally filtered out, can be used for energy harvesting at the relay. We derive the exact closed-form expression for the outage probability of this setup in terms of the bivariate Fox-H function. We also provide the asymptotic analysis to obtain the diversity order. Finally, Monte-Carlo simulations are carried out to verify the correctness of our analytical results.
\end{abstract}

Index Terms-energy harvesting, mixed free-space optical and radio-frequency (FSO-RF) system.

\section{INTRODUCTION}

As an efficient and highly secure technology, free-space optical (FSO) communication systems have attached extensive interests in academia [1]. Compared to radio frequency (RF) communications, FSO communications are capable of offering larger channel capacity to serve more users and developing a more reliable system.

However, the atmospheric turbulence may affect the correctness of the phase and intensity of the optical signal, which deteriorates the performance of the FSO link. Until now, several techniques have been proposed to effectively combat the atmospheric turbulence [1], such as error control coding, spatial diversity, and cooperative diversity. Among these techniques, relaying systems as a flexible and easydeployment technique can efficiently improve the reliability of the FSO communication networks. As such, mixed RF-FSO relaying transmission systems have gained great interests in the literature. The performance of mixed RF/FSO was studied in [2][3] and the references therein.

On the other hand, energy harvesting is a promising technique that can effectively solve the problems of the intermittent and scarce ambient energy, limited energy storage capacity, and the constrained devices in size and complexity. Up to now, the performance of different RF wireless systems with energy harvesting has been extensively studied in the literature [4]. However, to the best of the authors' knowledge, the performance of optical communication systems with energy harvesting only was reported in [5]-[9], for instance, optimal dual-hop visible light communication (VLC)-RF networks with energy harvesting [5], a secure hybrid VLC-RF system with light energy harvesting [6][7], hybrid RF-FSO systems with simultaneous wireless information and power transfer (SWIPT) [8], and the secure performance for a dual-hop mixed RF-FSO system [9]. However, the performance of mixed dual-hop FSO-RF systems with energy harvesting is still not reported in the literature.

In this work, we analyze the performance of a mixed FSORF relaying network with energy harvesting. The motivation behind such a mixed FSO-RF system can be explained as follows. Consider a downlink scenario that the source intends to communicate with the far RF users with the aid of a relay with both FSO and RF capabilities, where the source and the relay are installed at the top of two high-rise buildings and a FSO link is constructed between the source and the relay. In particular, there exists a connectivity gap between the backbone network and the last-mile access network, such as the housing estate, campus, and companies, and hence this last mile connectivity can be realized via high-speed FSO links. For such a mixed FSO-RF system, we assume that the direct current (DC) component of the optical signal which is generally filtered out in FSO communication systems can be collected and this extracted energy can be used to power information transmission. Therefore, our proposed scheme has its practical application since the DC component is reused and no additional power is required at the RF transmitter in the relay. With this setup, we develop a novel analytical framework to study the outage performance of the proposed system and provide both the exact result of the outage probability in terms of the bivariate Fox-H function and its asymptotic expression.

\section{System AND Channel Models}

Consider a dual-hop FSO-RF network with energy harvesting including one source (S) with FSO ability, one relay (R), and a destination (D). A block diagram of our system is shown in Fig. 1, where the hardware design framework of the energy harvesting is also presented. From practical considerations, we assume that the FSO link is impaired by Gamma-Gamma turbulence fading and pointing errors simultaneously. On the other hand, the RF link is assumed to suffer from Rayleigh fading due to the non light-of-sight propagation in urban environment. In addition, S, R, and D are assumed to be equipped with single aperture or antenna. We further assume that direct path between $\mathrm{S}$ and $\mathrm{D}$ is not available due to the long distance or blockage.

\section{A. First Hop FSO Transmission}

The subcarrier intensity modulation (SIM)-based binary phase shift keying (BPSK) scheme is adopted to convert the RF signal to an optical signal. More specifically, to ensure that the transmitted optical signal is positive, a DC bias $B$ is 


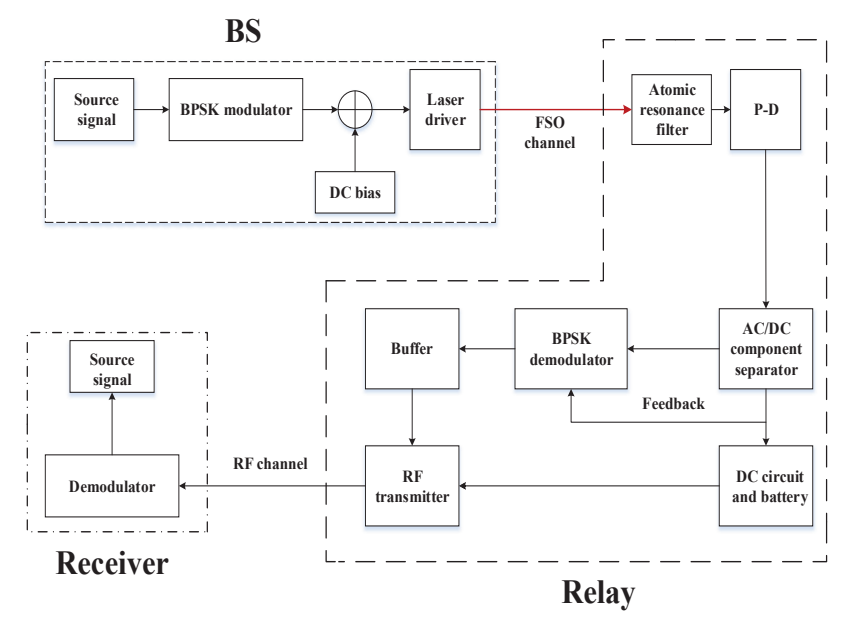

Fig. 1. Block diagram of dual-hop FSO-RF system with energy harvesting.

inserted to the electrical RF signal $R(t)$. Therefore, the optical signal at $\mathrm{S}$ can be written as $S(t)=\sqrt{P_{s}}[\zeta R(t)+B]$, where $P_{s}$ denotes the transmit power at $\mathrm{S}$ and $\zeta$ is the electricalto-optical conversion coefficient. Then, the resulting optical signal at the photo-detector (P-D) of R can be expressed as

$$
\begin{aligned}
& y_{1}(t)=h_{1} R_{\xi} A S(t)+n_{1}(t) \\
& =h_{1} R_{\xi} A \sqrt{P_{s}} \zeta R(t)+h_{1} R_{\xi} A \sqrt{P_{s}} B+n_{1}(t),
\end{aligned}
$$

where $R_{\xi}$ is the P-D responsivity and $A$ denotes the physical area of the P-D. Here, since we do not filter the DC component, ambient illumination may result in strong DC interference at P-D in daylight. Thus, we set that the elevation angle between the antennas of $\mathrm{S}$ and $\mathrm{R}$ is less than $30^{\circ}$. In this case, the effect of direct sunlight on FSO receiver can be significantly weakened. Moreover, we install an atomic resonance filter at the frontier of P-D to separate the ambient light that the polarization direction or the frequency is different from the source signal. Hence, the wideband shot noise raised by DC signal is restrained and $n_{1}(t)$ can be regarded as an additive white Gaussian noise (AWGN) with zero mean and variance $N_{01}$. In particular, the FSO channel gain can be expressed as $h_{1}=h_{a} h_{l} h_{p}$ [10], where $h_{a}$ is the atmospheric turbulence loss factor following Gamma-Gamma distribution, $h_{l}=\exp (-\sigma L)$ represents the path loss which is equal to a constant for given attenuation coefficient $\sigma$ and laser distance $L$, and $h_{p}$ is the pointing error loss factor. In [10], the authors used a simple Gaussian form to approximate $h_{p}$ as $h_{p} \approx A_{0} \exp \left(-\frac{2 r^{2}}{w_{z e q}^{2}}\right)$, where $A_{0}=[\operatorname{erf}(v)]^{2}, w_{z e q}^{2}=$ $w_{z}^{2} \frac{\sqrt{\pi} \operatorname{erf}(v)}{2 v \exp \left(-v^{2}\right)}, v=\frac{\sqrt{\pi} a}{\sqrt{2} w_{z}}$ is the ratio between aperture radius $a$ and beam waist $w_{z}$.

To ensure that the electrical signal $R(t)$ is within the linear region of the optical transmitter operation, we assume that the input electrical signal $\zeta R(t)$ varies around the DC bias $B$ with peak amplitude given in [5, eq. (2)]. Hence, the electrical-tooptical conversion coefficient can be expressed as

$$
\zeta= \begin{cases}\frac{B-I_{L}}{\mu}, & \text { if } B<\frac{I_{L}+I_{H}}{2} \\ \frac{I_{H}-B}{\mu}, & \text { if } B \geq \frac{I_{L}+I_{H}}{2}\end{cases}
$$

where $\mu$ is the electrical power of $R(t), I_{L}$ represents the minimum input bias current, and $I_{H}$ is the maximum input bias current.

\section{B. Energy Harvesting at the Relay}

With the help of an optical-to-electrical converter at the P$\mathrm{D}$, the optical signal is transformed into an electrical signal. Then, this electrical signal is separated into alternating current (AC) and DC components by using an AC/DC separator. To save the electrical power of the relay and make full use of the advantage of the FSO technique, we consider collecting the DC component $\eta h_{1} R_{\xi} A \sqrt{P_{s}} B$ which is generally filtered out as the transmit power at $\mathrm{R}$, where $\eta$ is the optical-toelectrical conversion coefficient. Simultaneously, the rest of the electrical signals (AC component $\eta h_{1} R_{\xi} A \sqrt{P_{s}} \zeta R(t)$ and the FSO channel noise) are modulated to the RF carrier by a BPSK modulator. Notice that the energy harvesting and information processing in the relay happen simultaneously. Hence, the total time of the signal transmission procedure is $T$ and the time of first-hop transmission and energy harvesting is $T / 2$, while the time of the second-hop transmission is $T / 2$. Therefore, the harvested energy at the relay can be written as [5][11]

$$
E_{h}=0.75 V_{t}\left(\eta h_{1} R_{\xi} A \sqrt{P_{s}} B\right)^{2} T / I_{0} .
$$

Then, this harvested energy can be applied as the transmit power of the relay, namely,

$$
P_{R}=\frac{E_{h}}{T / 2}=\frac{1.5 V_{t}\left(\eta h_{1} R_{\xi} A \sqrt{P_{s}} B\right)^{2}}{I_{0}},
$$

where $V_{t}$ is the thermal voltage $\left(V_{t} \approx 25 \mathrm{mVolt}\right)$ and $I_{0}$ is the dark saturation current of the P-D. With the amplifyand-forward assumption, we amplify the modulated electrical signal as $R_{1}(t)=G \sqrt{P_{R}} \eta\left[h_{1} R_{\xi} A \sqrt{P_{s}} \zeta R(t)+n_{1}(t)\right]$, where $G$ denotes the fixed amplifying gain.

\section{Second Hop RF Transmission}

At the second phase, the received signal at $\mathrm{D}$ can be expressed as

$$
y_{2}=h_{2} G \sqrt{P_{R}} \eta\left[h_{1} R_{\xi} A \sqrt{P_{s}} \zeta R(t)+n_{1}(t)\right]+n_{2}(t),
$$

where $h_{2}$ is the R-D channel gain obeying the Rayleigh distribution and $n_{2}(t)$ is the AWGN with zero mean and variance $N_{02}$. Therefore, we can obtain the overall instantaneous SNR as

$$
\gamma=\frac{\left|h_{2}\right|^{2} G^{2} \eta^{2} P_{R}\left(h_{1} R_{\xi} A \sqrt{P_{s}} \zeta\right)^{2}}{G^{2} P_{R} \eta^{2}\left|h_{2}\right|^{2} N_{01}+N_{02}}=\frac{\gamma_{1} \gamma_{2}}{\gamma_{2}+C},
$$

where $\gamma_{1}=\bar{\gamma}_{1}\left|h_{a}\right|^{2}\left|h_{p}\right|^{2}, \bar{\gamma}_{1}=\frac{\left(h_{l} R_{\xi} A \sqrt{P_{s}} \zeta\right)^{2}}{N_{01}}, \gamma_{2}=$ $\bar{\gamma}_{2}\left|h_{a}\right|^{2}\left|h_{p}\right|^{2}\left|h_{2}\right|^{2}, \bar{\gamma}_{2}=\frac{1.5 \eta^{2} V_{t}\left(\eta h_{l} R_{\xi} A \sqrt{P_{s} B}\right)^{2}}{I_{0} N_{02}}$, and $C=$ $\frac{1}{G^{2} N_{01}}$. To simplify the notation, we define $\rho_{i}=P_{s} / N_{0 i}$, $(i=1,2)$.

With the Gamma-Gamma fading and pointing error assumption, the composite probability density function (PDF) of $\gamma_{1}$ is given by [12]

$$
f_{\gamma_{1}}(x)=\frac{\xi^{2}}{2 x \Gamma(\alpha) \Gamma(\beta)} G_{1,3}^{3,0}\left(\frac{\alpha \beta}{A_{0}} \sqrt{\frac{x}{\bar{\gamma}_{1}}} \mid \begin{array}{c}
\xi^{2}+1 \\
\xi^{2}, \alpha, \beta
\end{array}\right),
$$




$$
I_{2}=\xi_{2} H_{2,0 ; 2,0 ; 3,1}^{0,1 ; 0,2 ; 0,3}\left[\begin{array}{l|l|l|l}
\frac{\gamma_{t h} \bar{\gamma}_{2}}{C \bar{\gamma}_{1}} \\
\frac{A_{0} \sqrt{\bar{\gamma}_{1}}}{\alpha \beta \sqrt{\gamma_{t h}}}
\end{array}\left|\begin{array}{l}
\left(1,-2, \frac{1}{2}\right),\left(1,1,-\frac{1}{2}\right) \\
-
\end{array}\right| \begin{array}{l}
(0,1),(1,1) \\
-
\end{array}\right]
$$

where $A_{0}$ is the fraction of the collected power, $\xi$ represents the ratio of the equivalent beam radius and jitter standard deviation at the P-D plane, and $G_{., .}(\cdot)$ denotes the Meijer's G-function [13, eq. (03.04.26.0008.01)]. Since the optical radiation follows plane wave propagation without inner scale, $\alpha$ and $\beta$ determined by atmospheric conditions can be written as [12] $\alpha=\left[\exp \left(\frac{0.49 \sigma_{R}^{2}}{\left(1+1.11 \sigma_{R}^{12 / 5}\right)^{7 / 6}}\right)-1\right]^{-1}$ and $\beta=\left[\exp \left(\frac{0.51 \sigma_{R}^{2}}{\left(1+0.69 \sigma_{R}^{12 / 5}\right)^{5 / 6}}\right)-1\right]^{-1}$, where $\sigma_{R}^{2}=$ $1.23 C_{n}^{2}\left(\frac{2 \pi}{\lambda}\right)^{7 / 6} L^{11 / 6}$ denotes the log irradiance variance, $C_{n}^{2}$ is the refractive index structure parameter, and $\lambda$ is the wavelength.

Then, by utilizing [13, 07.34.21.0002.01], the cumulative density function (CDF) of $\gamma_{1}$ can be expressed as

$$
F_{\gamma_{1}}(x)=\frac{\xi^{2}}{\Gamma(\alpha) \Gamma(\beta)} G_{2,4}^{3,1}\left(\frac{\alpha \beta}{A_{0}} \sqrt{\frac{x}{\bar{\gamma}_{1}}} \mid \begin{array}{c}
1, \xi^{2}+1 \\
\xi^{2}, \alpha, \beta, 0
\end{array}\right)
$$

Since the R-D link suffers from the Rayleigh fading, the CDF of $\left|h_{2}\right|^{2}$ can be obtained as

$$
F_{\left|h_{2}\right|^{2}}(x)=1-e^{-x}
$$

\section{Outage Performance Analysis}

\section{A. Exact Outage Probability Analysis}

In this section, the exact outage probability expression for our proposed mixed FSO-RF system is analyzed.

From (6), the overall outage probability can be written as

$$
\begin{aligned}
& P_{\text {OUT }}=P\left(\frac{\gamma_{1} \gamma_{2}}{\gamma_{2}+C}<\gamma_{t h}\right)=P\left(\frac{\bar{\gamma}_{1} X \bar{\gamma}_{2} X Y}{\bar{\gamma}_{2} X Y+C}<\gamma_{t h}\right) \\
& =P\left(\bar{\gamma}_{1} X<\gamma_{t h}\right)+P\left(Y<\frac{\gamma_{t h} C}{\bar{\gamma}_{1} \bar{\gamma}_{2} X^{2}-\gamma_{t h} \bar{\gamma}_{2} X}, \bar{\gamma}_{1} X>\gamma_{t h}\right) \\
& =F_{\gamma_{1}}\left(\gamma_{t h}\right)+I_{1} .
\end{aligned}
$$

where $X=\left|h_{a}\right|^{2}\left|h_{p}\right|^{2}, Y=\left|h_{2}\right|^{2}$, and $I_{1}$ is calculated as

$$
\begin{aligned}
& I_{1}=\int_{\frac{\gamma_{t h}}{\bar{\gamma}_{1}}}^{\infty}\left[1-\exp \left(-\frac{\gamma_{t h} C}{\left(\bar{\gamma}_{1} x-\gamma_{t h}\right) \bar{\gamma}_{2} x}\right)\right] \\
& \times \frac{\xi^{2}}{2 x \Gamma(\alpha) \Gamma(\beta)} G_{1,3}^{3,0}\left(\frac{\alpha \beta}{A_{0}} \sqrt{x} \mid \begin{array}{c}
\xi^{2}+1 \\
\xi^{2}, \alpha, \beta
\end{array}\right) d x .
\end{aligned}
$$

Generally, it is very difficult to evaluate the integral $I_{1}$ since the instantaneous SNR $\gamma$ involves $h_{1}^{4}$. By expressing $\exp (-x)$ in terms of Meijer's G-function [13, eq. (01.03.26.0004.01)] and let $z=\bar{\gamma}_{1} x-\gamma_{t h}$, (11) can be rewritten as

$$
\begin{aligned}
& I_{1}=1-F_{\gamma_{1}}\left(\gamma_{t h}\right)-\xi_{1} \int_{0}^{\infty}\left(\frac{z+\gamma_{t h}}{\bar{\gamma}_{1}}\right){ }^{-1} G_{0,1}^{1,0}\left(\left.\frac{C \gamma_{t h} \bar{\gamma}_{1}}{\bar{\gamma}_{2} z\left(z+\gamma_{t h}\right)}\right|_{0} ^{-}\right) \\
& \times G_{1,3}^{3,0}\left(\left.\frac{\alpha \beta}{A_{0}} \sqrt{\frac{z+\gamma_{t h}}{\bar{\gamma}_{1}}}\right|_{\xi^{2}, \alpha, \beta} ^{\xi^{2}+1}\right) d z \\
& =1-F_{\gamma_{1}}\left(\gamma_{t h}\right)-I_{2} .
\end{aligned}
$$

where $\xi_{1}=\frac{\xi^{2}}{2 \Gamma(\alpha) \Gamma(\beta)}$. By using the primary definition of the Meijer's G-function [13, eq. (07.34.02.0001.01)] and [14, eq. (3.194.3)], $I_{2}$ can be expressed as

$$
\begin{aligned}
& I_{2}=\frac{\xi_{2}}{(2 \pi i)^{2}} \int_{\ell_{1}} \int_{\ell_{2}} B\left(s+1, \frac{\omega}{2}-2 s\right) \Gamma(s) \Gamma(\omega+\alpha) \Gamma(\omega+\beta) \\
& \times \frac{\Gamma\left(\omega+\xi^{2}\right)}{\Gamma\left(\omega+\xi^{2}+1\right)}\left(\frac{C \bar{\gamma}_{1}}{\gamma_{t h} \bar{\gamma}_{2}}\right)^{-s}\left(\frac{\alpha \beta \sqrt{\gamma_{t h}}}{A_{0} \sqrt{\bar{\gamma}_{1}}}\right)^{-\omega} d s d \omega,
\end{aligned}
$$

where $\xi_{2}=\bar{\gamma}_{1} \xi_{1}, B(a, b)$ represents the beta function [14], and $\ell_{1}$ and $\ell_{2}$ are the integral contours in the complex domain. Furthermore, by applying [14, eq. (8.384.1)], (13) can be rewritten as

$$
\begin{aligned}
& I_{2}=\frac{\xi_{2}}{(2 \pi i)^{2}} \int_{\ell_{1}} \int_{\ell_{2}} \frac{\Gamma\left(\frac{\omega}{2}-2 s\right)}{\Gamma\left(\frac{\omega}{2}-s+1\right)} \Gamma(s) \Gamma(s+1)\left(\frac{C \bar{\gamma}_{1}}{\gamma_{t h} \bar{\gamma}_{2}}\right)^{-s} \\
& \times \frac{\Gamma\left(\omega+\xi^{2}\right) \Gamma(\omega+\alpha) \Gamma(\omega+\beta)}{\Gamma\left(\omega+\xi^{2}+1\right)}\left(\frac{\alpha \beta \sqrt{\gamma_{t h}}}{A_{0} \sqrt{\bar{\gamma}_{1}}}\right)^{-\omega} d s d \omega .
\end{aligned}
$$

Finally, according to the definition of the bivariate Fox-H function [15, eq. (2.55)], $I_{2}$ can be obtained at the top of this page. From (10), (12), and (15), the overall outage probability can be expressed as

$$
P_{O U T}=1-I_{2}
$$

\section{B. Asymptotic Analysis}

Since the exact outage probability expression (16) involves the bivariate Fox-H function, it can not provide any explicit insights. Therefore, we develop an asymptotic analysis to observe the diversity order. By utilizing the approximate method [16], at the high SNR region, the overall outage probability has the lower bound as

$$
\begin{aligned}
& P\left(\frac{\gamma_{1} \gamma_{2}}{\gamma_{2}+C}<\gamma_{t h}\right) \geq 1-P\left(X>\frac{\gamma_{t h}}{\bar{\gamma}_{1}}, Y>\frac{\gamma_{t h} C}{\bar{\gamma}_{1} \bar{\gamma}_{2} X^{2}}\right) \\
& =1-\int_{\frac{\gamma_{t h}}{\bar{\gamma}_{1}}}^{\infty} \exp \left(-\frac{\gamma_{t h} C}{\bar{\gamma}_{1} \bar{\gamma}_{2} x^{2}}\right) \\
& \times \frac{\xi^{2}}{2 x \Gamma(\alpha) \Gamma(\beta)} G_{1,3}^{3,0}\left(\frac{\alpha \beta}{A_{0}} \sqrt{x} \mid \begin{array}{c}
\xi^{2}+1 \\
\xi^{2}, \alpha, \beta
\end{array}\right) d x .
\end{aligned}
$$

By using the fact $1-e^{-x} \sim x$ and applying [13, eq. (07.34.21.0085.01)], (17) can be rewritten as

$$
\begin{aligned}
& P_{\text {OUT }} \approx \frac{\xi^{2}}{\Gamma(\alpha) \Gamma(\beta)} G_{2,4}^{3,1}\left(\frac{\alpha \beta}{A_{0}} \sqrt{\frac{\gamma_{t h}}{\bar{\gamma}_{1}}} \mid \begin{array}{c}
1, \xi^{2}+1 \\
\xi^{2}, \alpha, \beta, 0
\end{array}\right) \\
& +\frac{C \bar{\gamma}_{1} \xi^{2}}{\gamma_{t h} \bar{\gamma}_{2} \Gamma(\alpha) \Gamma(\beta)} G_{2,4}^{4,0}\left(\frac{\alpha \beta}{A_{0}} \sqrt{\frac{\gamma_{t h}}{\bar{\gamma}_{1}}} \mid \begin{array}{l}
\xi^{2}+1,5 \\
4, \xi^{2}, \alpha, \beta
\end{array}\right) .
\end{aligned}
$$


Using [13, eq. (07.34.06.0006.01)], we obtain the asymptotic expression as

$$
\begin{aligned}
& P_{\text {OUT }}^{A s y m} \rightarrow \frac{\xi^{2}}{\Gamma(\alpha) \Gamma(\beta)}\left[\sum_{k=1}^{3} \frac{\prod_{j=1, j \neq k}^{3} \Gamma\left(b_{j}-b_{k}\right) \Gamma\left(b_{k}\right)}{\Gamma\left(\xi^{2}+1-b_{k}\right) \Gamma\left(1+b_{k}\right)}\right. \\
& \left.\times\left(\frac{\alpha \beta}{A_{0}} \sqrt{\frac{\gamma_{t h}}{\bar{\gamma}_{1}}}\right)^{b_{k}}\right]+\frac{C \bar{\gamma}_{1} \xi^{2}}{\gamma_{t h} \bar{\gamma}_{2} \Gamma(\alpha) \Gamma(\beta)} \\
& \times \sum_{K=1}^{4} \frac{\prod_{J=1, J \neq K}^{4} \Gamma\left(B_{J}-B_{K}\right)}{\Gamma\left(\xi^{2}+1-B_{K}\right) \Gamma\left(5-B_{K}\right)}\left(\frac{\alpha \beta}{A_{0}} \sqrt{\frac{\gamma_{t h}}{\bar{\gamma}_{1}}}\right)^{B_{K}},
\end{aligned}
$$

where $b_{k}=\left(\xi^{2}, \alpha, \beta, 0\right)$ and $B_{K}=\left(\xi^{2}, \alpha, \beta, 4\right)$.

Remark 1: Let $\bar{\gamma}_{1} \rightarrow \infty$ and $\bar{\gamma}_{2}=\bar{\gamma}_{1}^{a}$. Then, the diversity order can be formulated as

$$
\begin{aligned}
d & =-\lim _{\rho \rightarrow \infty} \frac{\log \left(P_{\text {OUT }}^{\text {Asym }}\right)}{\log (\rho)}=\min \left\{\frac{b_{k}}{2}, \frac{B_{K}}{2}+a-1\right\} \\
& =\min \left\{\frac{\xi^{2}}{2}, \frac{\alpha}{2}, \frac{\beta}{2}, a+1, \frac{\xi^{2}}{2}+a-1, \frac{\alpha}{2}+a-1, \frac{\beta}{2}+a-1\right\} .
\end{aligned}
$$

Above expression indicates that the diversity order of our proposed system is determined by the values of $a=\log _{\bar{\gamma}_{1}} \bar{\gamma}_{2}$, $\xi, \alpha$, and $\beta$. When $a \geq 1$, i.e. $\bar{\gamma}_{2} \geq \bar{\gamma}_{1}, d$ is equal to $\min \left\{\xi^{2} / 2, \alpha / 2, \beta / 2, a+1\right\}$. From (19), the term including $\bar{\gamma}_{2}$ can be omitted for large $\bar{\gamma}_{2}$.

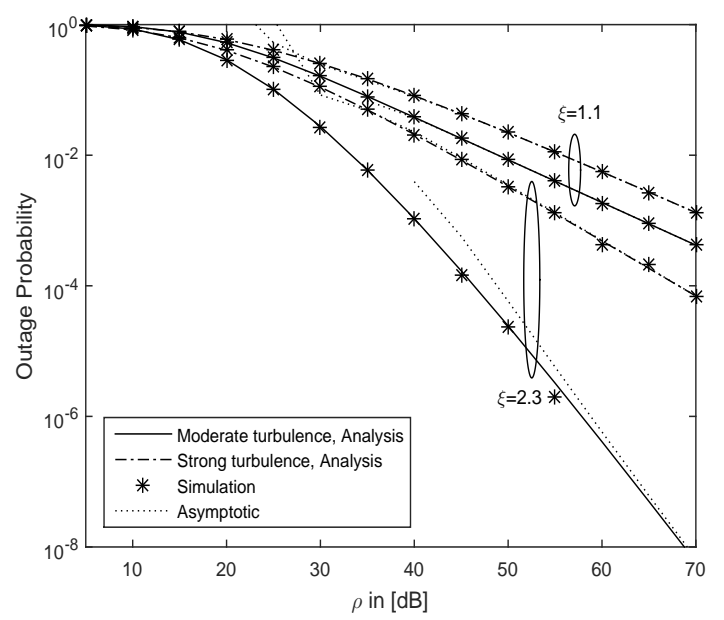

Fig. 2. Outage probability versus $\rho$ for different $\xi$ under moderate and strong atmospheric turbulence parameters. $\left(B=30 \mathrm{~mA}, R_{\xi}=0.6 \mathrm{~A} / \mathrm{W}\right.$ and $\eta=$ $0.6)$.

\section{NumericAl Results}

In this section, we present selected numerical results to show the outage performance of the mixed FSO-RF system with energy harvesting. Notice that there is no internal function available in current computing software to evaluate the bivariate Fox-H function. In [17][18], the authors proposed a MATLAB implementation method to evaluate the bivariate Fox-H function. In this work, we use this method to implement the numerical root finding. According to [10], we set $(\alpha, \beta)=(5.41,3.78)$ and $(3.99,1.70)$ corresponding to

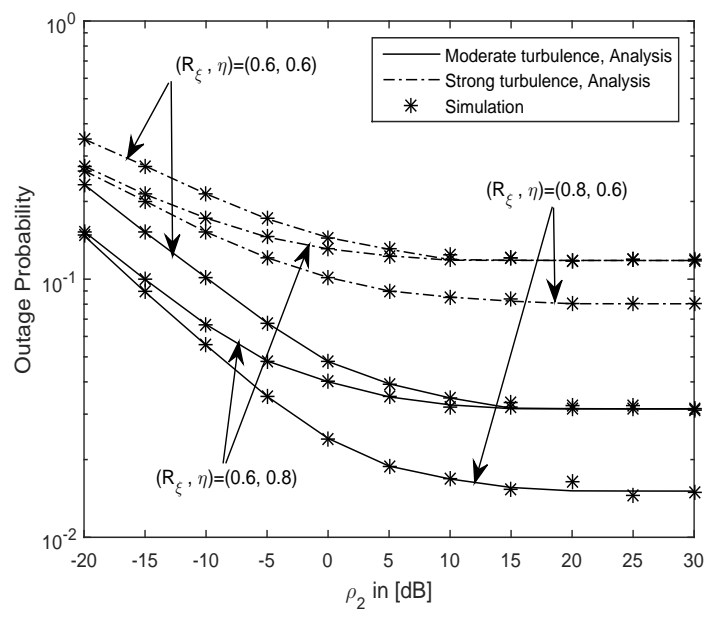

Fig. 3. Outage probability versus $\rho_{2}$ for different $R_{\xi}$ and $\eta$ under different atmospheric turbulence conditions. ( $\rho_{1}=30 \mathrm{~dB}, \xi=2.3$, and $B=10 \mathrm{~mA}$ ).

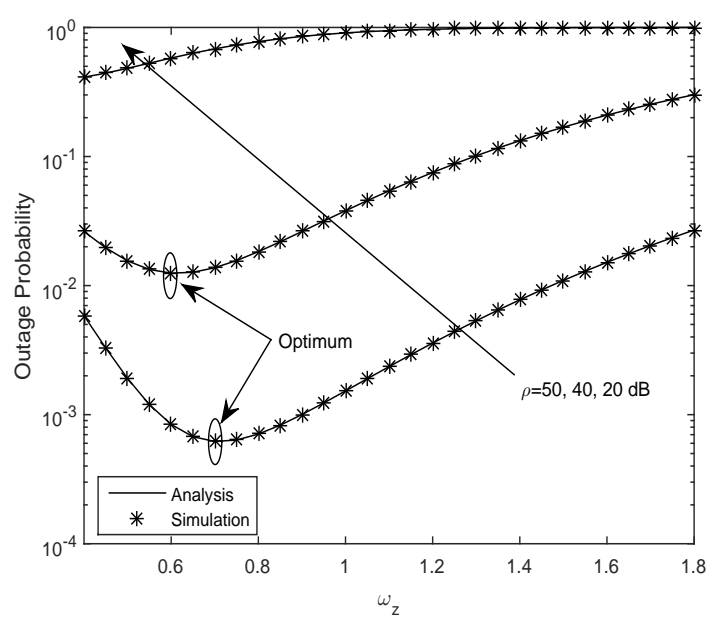

Fig. 4. Outage probability for different values of $\rho$ versus $w_{z}$. ( $\rho_{1}=\rho_{2}=\rho$, $\xi=2.3, B=10 \mathrm{~mA}, R_{\xi}=0.8 \mathrm{~A} / \mathrm{W}$ and $\eta=0.8$ ).

the moderate turbulence and strong turbulence, respectively and $h_{l}=0.9$. Additionally, in the following figures, we set $A=0.1 \mathrm{~m}^{2}, G=1, I_{0}=10^{-10}$ Ampere, and $\gamma_{t h}=\exp (0.1)$. In Fig. 2-Fig. 4, Monte-Carlo simulations perfectly match with the theoretical results, which proves the accuracy of our derivations.

In Fig. 2, we assume $\rho_{1}=\rho_{2}=\rho$ and plot the outage probability in (16) versus $\rho$ for different values of $\xi$. It can be clearly observed that the curves of the outage probability have the same slopes when $\xi=1.1$ even if under the different turbulence conditions, which means that these curves have the same diversity order. However, when the value of $\xi$ is equal to 2.3 , we can see that the slopes of the curves are different as changing the turbulence conditions. Generally, we set the thermal voltage $V_{t} \approx 25 \mathrm{mVolt}$ and the dark saturation current of the P-D, $I_{0}$, is in the order of $10^{-12} \sim 10^{-9}$. In this case, according to Remark 1 , we can obtain $a \geq 1$ and $d=\min \left\{\xi^{2} / 2, \alpha / 2, \beta / 2, a+1\right\}$. Thus, it can be seen that the curves corresponding to $\xi=1.1$ and $\xi=2.3$ have the same 
and different slopes, respectively. In other words, this also verifies the correctness of Remark 1. Furthermore, it can be observed that increasing the value of $\xi$ significantly improves the outage performance since the larger the value of $\xi$, the smaller the value of jitter, and the weaker the impact of the pointing error on FSO channel.

Fig. 3 demonstrates the outage probability versus $\rho_{2}$ for different values of $R_{\xi}$ and $\eta$ under moderate and strong turbulence conditions. For fixed $\rho_{1}$, we can see that the outage probability is independent of $\rho_{2}$ at high $\rho_{2}$. The reason is that the term including $\bar{\gamma}_{2}$ in (10) can be omitted for large $\bar{\gamma}_{2}$ and the outage probability is only related to $\bar{\gamma}_{1}$. Besides, from Fig. 3, it can be seen that as the outage probability is a function of $\rho_{2}$, an error floor appears when $\rho_{2} \geq 10 \mathrm{~dB}$. In other words, the resulting average SNR of the RF link, $\bar{\gamma}_{2}$, is large, which means that the harvested energy in our scheme can ensure the information transmission in the second RF hop. On the other hand, from (3), $R_{\xi}$ and $\eta$ are the main factors affecting the FSO energy harvesting efficiency and increasing both of them can improve our system performance. Notice that increasing the value of $\eta$ hardly enhances the outage performance when $\rho_{2} \geq 10 \mathrm{~dB}$ since it is a parameter of $\bar{\gamma}_{2}$. In addition, it can be observed that increasing $R_{\xi}$ can obtain a better outage performance than increasing $\eta$, which can be explained that both $\bar{\gamma}_{1}$ and $\bar{\gamma}_{2}$ involve the term $R_{\xi}$. Accordingly, in comparison to $\eta, R_{\xi}$ has more significant effect on our FSO-based energy harvesting system.

To demonstrate the effect of misalignment errors on the outage performance of our system, we depict the outage probability under moderate turbulence condition versus $w_{z}$ and compare with different SNR cases in Fig. 4 . When $\rho=40,50$ $\mathrm{dB}$, we can observe that increasing $w_{z}$ causes that the outage probability first decreases and then increases. The primary reason is that increasing $w_{z}$ decreases the value of $A_{0}$ but increases the value of $w_{z e q}^{2}$, and in turn generates two converse effects on $h_{p}$. However, for $\rho=20 \mathrm{~dB}$, increasing $w_{z}$ does not appear an optimum point on the curve but continuously impairs the outage performance. This is because when the resulting $\bar{\gamma}_{1}$ is small, the term $\bar{\gamma}_{1} A_{0}$, compared to $w_{z e q}^{2}$, has smaller impact on $\gamma_{1}$, and then the value of $\gamma_{1}$ is almost only influenced by $w_{z e q}^{2}$.

\section{Conclusions}

In this work, we designed a novel FSO-based energy harvesting system and analyzed the outage performance for the mixed FSO-RF network adopting this energy harvesting scheme, where the pointing error loss on FSO link was taken into account. More concretely, we derived an exact expression of the outage probability in terms of the bivariate Fox- $\mathrm{H}$ function and presented the diversity order analysis. Compared with the traditional RF-based energy harvesting scheme, our proposed FSO-based energy harvesting systems have higher and more controllable energy efficiency, which can be realized by changing the P-D responsivity and the physical area of the P-D. Here, we only investigate the relationship between outage performance and $R_{\xi}$ since the impact of $R_{\xi}$ on $\gamma$ is the same as that of $A$. Results shows that a larger $R_{\xi}$ results in a better performance and, compare with a larger $\eta$, has a more significant effect on the outage probability, which means that the energy harvesting efficiency can be controlled artificially. Additionally, according to the results in asymptotic analysis, the diversity order of our system only depends on the values of $\alpha, \beta, a$ and $\xi$. Finally, the expression of $h_{p}$ indicates that there exist two opposite effects on pointing errors when increasing the value of $w_{z}$. Thus, we can find an optimal $w_{z}$ to balance these two effects and further optimize outage performance.

\section{REFERENCES}

[1] M. A. Khalihi, and M. Uysal, "Survey on free space optical communication: a communication theory perspective," IEEE Commun. Surv. Tutor. vol. 5, no. 11, pp. 1139-1144, 2014.

[2] L. Yang, M. O. Hasna and I. S. Ansari, "Unified performance analysis for multiuser mixed $\eta-\mu$ and $\mathcal{M}$-distribution dual-hop RF/FSO systems," IEEE Trans. Commun., vol. 65, no. 8, pp. 3601-3613, Aug. 2017

[3] L. Yang, T. Liu, J. Chen, and M.-S. Alouini, "Physical-Layer Security for Mixed $\eta-\mu$ and $\mathcal{M}$-Distribution Dual-Hop RF/FSO Systems," IEEE Trans. Veh. Technol., DOI: 10.1109/TVT.2018.2877136, Oct. 2018.

[4] J. Ren, et al., "RF energy harvesting and transfer in cognitive radio sensor networks: opportunities and challenges," IEEE Commun. Mag., vol. 56, no. 1, pp. 104-110, Jan. 2018.

[5] T. Rakia, H. C. Yang, F. Gebali, M. S. Alouini, "Optimal design of dual-hop VLC/RF communication system with energy harvesting," IEEE Commun. Lett., vol. 20, no. 10, pp. 1979-1982, Oct. 2016.

[6] G. F. Pan, J. Ye, Z. G. Ding, "Secure hybrid VLC-RF systems with light energy harvesting," IEEE Trans. Commun., vol. 65, no. 10, pp.43484359, Oct. 2017.

[7] Z. Liao, L. Yang, J. Chen, H.-C. Yang, and M.-S. Alouini, "Physical Layer Security for Dual-Hop VLC/RF Communication Systems," IEEE Wireless Commun. Lett., DOI: 10.1109/LCOMM.2018.2873725, Oct. 2018.

[8] B. Makki, T. Svensson, K. Buisman, J. Perez, and M. S. Alouini, "Wireless energy and information transmission in FSO and RF-FSO links," IEEE Wireless Commun. Lett., vol. 7, no. 1, pp. 90-93, Feb. 2018.

[9] H. Lei, Z. Dai, K.-H. Park, W. Lei, G. Pan, and M. S. Alouini, "Secrecy outage analysis of mixed RF-FSO downlink SWIPT systems," IEEE Trans. Commun., DOI: 10.1109/TCOMM.2018.2865944, 2018.

[10] A. A. Farid and S. Hranilovic, "Outage capacity optimization for freespace optical links with pointing errors," J. Lightw. Technol., vol. 25, no. 7, pp. 1702-1710, Jul. 2007.

[11] C. Li, W. Jia, Q. Tao, and M. Sun, Solar cell phone charger performance in indoor environment, in Proc. IEEE 37th Annu. Northeast Bioeng. Conf. (NEBEC), Troy, NY, USA, Apr. 2011, pp. 1-2.

[12] H. E. Nistazakis, E. A. karagianni, A. D. Tsigopoulos, M. E. Fafalios, and G. S. Tombras, "Average capacity of optical wireless communication systems over atmospheric turbulence channels," J. Lightw. Technol., vol. 27, no. 8, pp. 974-978, Apr. 2009.

[13] Wolfram, The Wolfram functions site, Available: http://functions.wolfram.com.

[14] I. S. Gradshteyn and I. M. Ryzhik, Table of Integrals, Series and Products, 7th ed. San Diego, CA, USA: Academic, 2007.

[15] A.M. Mathai, R.K. Saxena, and H.J. Haubold, The H-Function: Theory and Applications, Springer, New York, 2010.

[16] J. N. Laneman, D.N.C. Tse and G. W. Wornell, "Cooperative diversity in wireless networks: efficient protocols and outage behavior," IEEE Trans. Inf. Theory, vol. 50, no. 12, pp. 3062-3080, Dec. 2004.

[17] K. P. Peppas, "A new formula for the average bit error probability of dual-hop amplify-and-forward relaying systems over generalized shadowed fading channels," IEEE Wireless Commun. Lett., vol. 1, no. 2, pp. 85-88, Apr. 2012.

[18] H. Chergui, M. Benjillali and M.-S. Alouini, "Rician $K$-factor-based analysis of XLOS service probability in 5G outdoor ultra-dense networks," 2018. [Online]. Available: arxiv.org/pdf/1804.08101v2. 